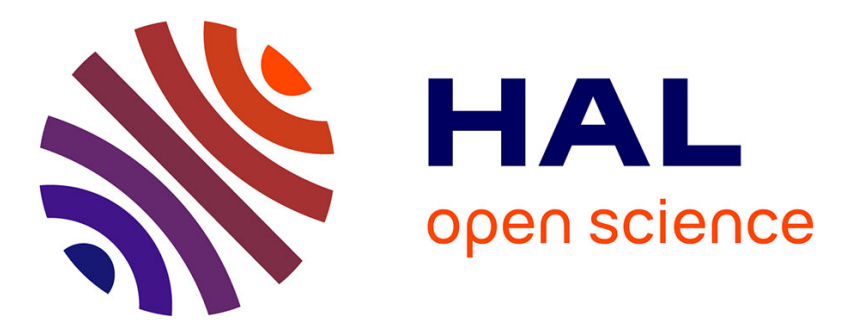

\title{
Alternative Parent Selection for Multi-Path RPL Networks
}

Tomas Lagos Jenschke, Georgios Papadopoulos, Remous-Aris Koutsiamanis, Nicolas Montavont

\section{- To cite this version:}

Tomas Lagos Jenschke, Georgios Papadopoulos, Remous-Aris Koutsiamanis, Nicolas Montavont. Alternative Parent Selection for Multi-Path RPL Networks. 2019 IEEE 5th World Forum on Internet of Things (WF-IoT'19), Apr 2019, Limerick, Ireland. pp.533-538, 10.1109/WF-IoT.2019.8767236 . hal-02268764

\section{HAL Id: hal-02268764 \\ https://hal.science/hal-02268764}

Submitted on 3 Mar 2020

HAL is a multi-disciplinary open access archive for the deposit and dissemination of scientific research documents, whether they are published or not. The documents may come from teaching and research institutions in France or abroad, or from public or private research centers.
L'archive ouverte pluridisciplinaire HAL, est destinée au dépôt et à la diffusion de documents scientifiques de niveau recherche, publiés ou non, émanant des établissements d'enseignement et de recherche français ou étrangers, des laboratoires publics ou privés. 
archives-ouvertes

\title{
Alternative Parent Selection for Multi-Path RPL Networks
}

\author{
Tomas Lagos Jenschke, Tomas Jenschke, Tomas Lagos Jenschke, Georgios \\ Papadopoulos, Remous-Aris Koutsiamanis, Nicolas Montavont
}

\section{- To cite this version:}

Tomas Lagos Jenschke, Tomas Jenschke, Tomas Lagos Jenschke, Georgios Papadopoulos, RemousAris Koutsiamanis, et al.. Alternative Parent Selection for Multi-Path RPL Networks. 2019 IEEE 5th World Forum on Internet of Things (WF-IoT'19), Apr 2019, Limerick, Ireland. pp.533-538, 10.1109/WF-IoT.2019.8767236 . hal-02268764

\section{HAL Id: hal-02268764 \\ https://hal.archives-ouvertes.fr/hal-02268764}

Submitted on 3 Mar 2020

HAL is a multi-disciplinary open access archive for the deposit and dissemination of scientific research documents, whether they are published or not. The documents may come from teaching and research institutions in France or abroad, or from public or private research centers.
L'archive ouverte pluridisciplinaire HAL, est destinée au dépôt et à la diffusion de documents scientifiques de niveau recherche, publiés ou non, émanant des établissements d'enseignement et de recherche français ou étrangers, des laboratoires publics ou privés. 


\title{
Alternative Parent Selection for Multi-Path RPL Networks
}

\author{
Tomas Lagos Jenschke, Georgios Z. Papadopoulos, Remous-Aris Koutsiamanis and Nicolas Montavont \\ IMT Atlantique, Irisa, UBL, France \\ Email: \{firstname.lastname\}@imt-atlantique.fr
}

\begin{abstract}
The IPv6 Routing Protocol for Low-Power and Lossy Networks (RPL) is designed for Internet of Things (IoT) applications. This Low Power and Lossy Network (LLN) protocol builds a Destination Oriented Directed Acyclic Graph (DODAG) network by transmitting DODAG Information Object (DIO) control packets. The DODAG structure created is hierarchical, where the upward routes are towards nodes with a lower rank, with the sink/root node having the lowest rank. To achieve connectivity, RPL nodes select their routes through an Objective Function (OF). To improve reliability and minimize jitter, we implemented Packet Replication and Elimination (PRE) and a multi-path routing OF. However, no standardized algorithm exists for this purpose although for PRE, several algorithms have been proposed for selecting an Alternative Parent (AP) node. An example of this is the LeapFrog Collaboration (LFC) algorithm, which selects an AP using a triangular pattern. In this paper, we examine how this selection affects power, network overload, delay and jitter. We focus on analyzing the selection of an AP, in terms of how strict the selection should be when a triangular pattern is used and what trade-offs are presented between network traffic and reliability.

Index Terms-Multi-path, RPL, LeapFrog Collaboration, PRE, Determinism, LLN, IEEE 802.15.4 - TSCH
\end{abstract}

\section{INTRODUCTION}

Industry 4.0 has been a rapidly developing field in recent years. The aim has been to reach simple, low cost, and efficient $\mathrm{p}$ roduction. I $\mathrm{n}$ this i ndustry, i ntelligent $\mathrm{f}$ actories are envisioned, which employ controlled production to achieve this goal. During the development of this industry, the deployment of Internet of Things (IoT) devices has increased exponentially as an essential part of the production chain.

However, due to the different applications that must be supported, a higher quality of service is required, which typically IoT technologies do not provide. An example of this is the reliability and determinism that must be maintained in an industrial wireless network.

Throughout the use of Low Power and Lossy Network (LLN) devices, different protocols have been proposed. The IPv6 Routing Protocol for Low-Power and Lossy Networks (RPL) [11] forms a hierarchical network between nodes and is characterized by its routing adaptivity in wireless networks. This protocol can select one or more routes per node. The selection of a single route will depend on the Objective Function (OF) in a Destination Oriented Directed Acyclic Graph (DODAG), while the selection of multiple routes does not have an established way of being made. However, its implementation allows parallel transmissions, improving delivery reliability.

The problem lies in the trade-off presented when multipath routing is used. To ensure a high Packet Delivery Ratio (PDR), it is necessary to transmit a higher number of packet copies. However, a higher number of copies also leads to higher power consumption. In this paper, we focus on the performance evaluation of multi-path routing by selecting an Alternative Parent (AP) using the Triangular Pattern [7] and LeapFrog Collaboration (LFC) [4], [8]. In addition, the Soft Common Ancestor (CA) algorithm was added as the logical extension of the previous two.

\section{BACKGROUND AND RELATED WORK}

\section{A. Background}

1) IEEE 802.15.4-TSCH: Time-Slotted Channel Hopping (TSCH) [1] is a technique that uses the channel hopping scheme with the main objective of reducing the impact of external interference. TSCH is responsible for activity scheduling within the Medium Access Control (MAC) layer of each node, synchronizing the transmission and reception of data between nodes as well as their inactivity. Time in a TSCH network is quantized into distinct and consecutive periods with the same duration, called timeslots. TSCH networks also support multiple communication channels, which are abstracted into channel offsets. Each action that a node takes is directly related to a cell (a pair of a timeslot and a channel offset), multiple of which are grouped into slotframes of fixed size. These slotframes repeat during the life of the network. Currently IEEE 802.15.4-TSCH describes the general form and function of TSCH schedules, but the exact structure is free to be defined by the implementation based on the specific needs of the application domain.

2) RPL: A distance-vector routing protocol [11], which aims to generate routing links between LLN devices. Within RPL there is a coordinating node called the DODAG root. This node has all the necessary information to incorporate different devices into the network to which it belongs.

Each set of nodes within a network is part of one or more Directed Acyclic Graph (DAG)s. In each DAG, once the root node is defined, the DAG will be oriented to it, generating a DODAG. 
RPL uses the following types of control messages:

- DODAG Information Object (DIO): It carries the necessary information so that a device can be incorporated into the DODAG. It is broadcast to all the neighbors that a node has.

- Destination Advertisement Object (DAO): It allows the construction of downward routes (from the root towards the leaves). It is transmitted by a node when it has added a parent node from its Parent Set (PS).

- DODAG Informational Solicitation (DIS): It is used to explicitly request a DIO as a response. It is also used to identify the status of its neighborhood.

RPL also supports the following downward traffic operation modes:

- Storing mode: This method provides parents with the information about their children to achieve the downstream transmission. The DAO packets will be transmitted to the parents selected by the nodes instead of the DODAG root.

- Non-storing mode: This method provides the DODAG root with the routing table for downward transmissions. To achieve this, the DAO packets are transmitted directly to the DODAG root.

For the selection of routes in RPL, the OF is used. This function uses certain parameters which allow a node to select a specific route depending on the target. These parameters are defined in metrics like the ones shown below:

- Hop Counting (HC): This metric provides the number of hops that a packet needs to reach its destination. This ensures the shortest route but not the most reliable route.

- Expected Transmission Count (ETX): The use of this metric is to find the best route according to the link quality. This quality is based on the probability of a successful transmission. Therefore, it does not optimize the distance, but rather its probability of success.

3) 6TiSCH: A working group established in the Internet Engineering Task Force (IETF) [10] which is focused on the communication between the IEEE 802.15.4-TSCH and the upper layers such as the IPv6 over Low power Wireless Personal Area Networks (6LoWPAN) layer, RPL and the Constrained Application Protocol (CoAP). An important part of IPv6 over the TSCH mode of IEEE 802.15.4e (6TiSCH) is scheduling, i.e., the process of deciding on and creating the TSCH schedule in use. For this purpose there are two broad categories of scheduling: centralized, where the schedule for all of the network nodes is created, and decentralized where the schedule is created locally in different parts of the network. In the rest of this work we assume the use of centralized scheduling, which defines the cells for the transmission and reception of both data and control packets.

4) Packet Replication and Elimination (PRE): PRE [9] generates copies of a single data packet to increase the probability that it can reach its destination. The copies are transmitted in parallel along different routes, so that if one of these fails in its transmission, there is a probability that the second route will deliver the message. In this way the reliability of the transmission improves the reception of the packet. This is especially important for high priority data.

PRE controls the additional traffic of replicated packets by removing the copies that subsequently arrive at a node. This means that the node will only receive or forward a single data packet while dropping the rest of the subsequent copies.

\section{B. Related Work}

In [6], the authors propose three scheme types, based on an Energy-awareness Load Balancing (ELB) protocol, a Fast Local Repair protocol (FLR), and one on a combination of both (ELB-FLR). The schemes are implemented by different OFs. ELB is based on a combination of the hop counting metric and residual energy. A node calculates its rank by dividing the number of total hops with the maximum number of hops plus the remaining energy of the node. The resulting value is added to a list of increasing ranks where the Preferred Parent (PP) is the first node in the list and the AP the following node. In the case of FLR, the focus is on the increase of path redundancy for high priority situations. For this, the authors propose the use of a sibling node (same rank) as a possible AP.

In [7] the authors propose and analyze three different types of patterns: "Disjoint", "Triangle" and "Braided". Each pattern is intended to increase the reliability of the communication by using an alternative route in addition to the preferred route. This results in a greater number of opportunities for a packet to reach the destination. Depending on the algorithm, the number of nodes that will forward the packet will be affected, which results in higher power consumption and increased traffic overhead.

In [3], the authors propose the implementation of an Expected Lifetime (ELT) metric to maximize the useful life of the most restricted nodes. The PP will be chosen if this parent maximizes the life of the nodes that have the lowest residual energy and the AP will be chosen by the ETX metric.

\section{Problem Statement}

The authors in [4], [8] propose the LFC algorithm which selects an AP by the looking for a common ancestor between the Preferred Parent (PP) and a possible node candidate. However, these works focus on designing an algorithm which achieves its determinism goals, and to do so a simpler network topology and some simplifying assumptions are used. As a result, the consequences of using this algorithm in a more complicated environment are not addressed. In this section we analyze the results of this algorithm when in different scenarios that were missing or that were not taken into consideration.

\section{A. Topology}

In [4], [8] a topology is used in which all the nodes, except the direct children of the root, have two nodes in their PS. It should be mentioned that for each hop there are two nodes, and therefore, each pair of nodes per hop layer will have the same PS. Due to the structure of this topology, it is guaranteed that each node has an AP. The problem lies in the situation where there are more than two nodes in the PS. 


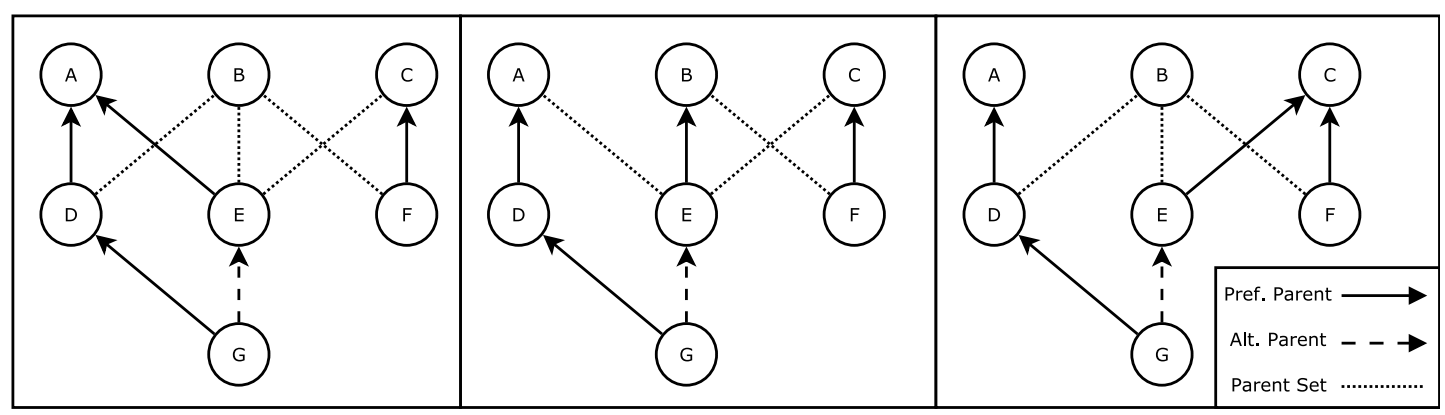

(a) Strict CA.

(b) Medium CA.

(c) Soft CA.

Fig. 1: AP selection: Strict triangular (Figure 1a), Medium triangular (Figure 1b), Soft triangular (Figure 1c).

A node in LFC selects an AP if the PS of the candidate AP contains the PP of the node's PP, as shown in Figure 1b. In the case of a wide topology an AP can isolate itself from its target area and, in turn, replicate to its distinct PS, covering a larger number of nodes and therefore creating a larger number of copies.

\section{B. Flooding}

LFC increases its probability of successful delivery by increasing the number of copies that a node transmits and receives. This number of copies are affected by:

- Number of Re-Transmissions (RTXs): For each unacknowledged transmission, the node will make a new attempt, with a maximum of 2 total attempts $(1 \mathrm{TX}+$ 1 RTX).

- Overhearing: Due to the nature of wireless networks, nodes within the range of a radio transmission can listen to the message. This means that a node can receive the message without it being the intended recipient. However, LFC defines that only the nodes that are in the parent set of the source node should be able to forward the packet.

These methods taken together lead to increased reliability but also to flooding due to multiple packet copies being forwarded through the network.

\section{Energy consumption}

Energy consumption is directly related to the topology and the flooding produced by the packet copies. The greater the number of copies transmitted, the longer the time a node will be active. At the same time, a greater number of traversed nodes leads to a smaller number of idling nodes. Therefore, it is very important to achieve a trade-off between the number of packets transmitted versus the number of nodes in use.

\section{Contributions \\ TO THE COMMON ANCESTOR Algorithm}

\section{A. Topology}

To reduce the effects of randomness and to aid the analytical description of the behavior of the network we chose a topology for the network as illustrated in Figure 2. The network consists of a source node $S$, a root/sink node $R$ and $L$ layers of nodes with $N$ nodes each, i.e.

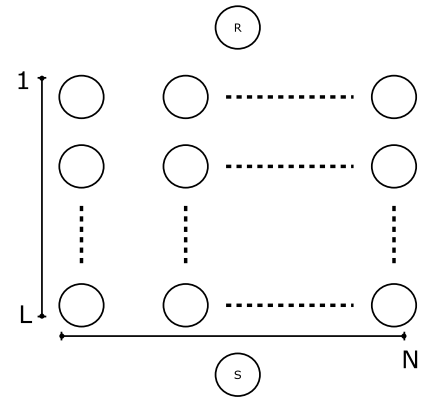

Fig. 2: Network topology.

$|\mathcal{L}(i)|=N, i \in\{1,2, \cdots, L\}$ where $\mathcal{L}(i)$ is the set of nodes in the $i^{\text {th }}$ layer. The $S$ node and the nodes in layers $2 \cdots L$ each have all the $N$ nodes in the layer above them in their parent set $(P S)$, i.e. $P S(S)=\mathcal{L}(L),|P S(S)|=N$ and $\forall i \in\{2, \cdots, L\} . \forall n \in \mathcal{L}(i) . P S(n)=\mathcal{L}(i-1),|P S(n)|=N$. The nodes in layer 1 only have the root node $\mathrm{R}$ in their parent set. Finally, the nodes report a subset of their parent set in the PS extension [5] of the Node State and Attribute (NSA) object in the Metric Container (MC) of DIO messages (referred to as the $P S_{M C}$ parent set here on). This $P S_{M C}$ extension contains a fixed number of addresses $M$, with $1 \leq M \leq N$.

In this work, we define three methods of selecting an AP through common ancestors, ranging from strict to relaxed selection criteria. In the following we calculate the probability of selecting an AP for node $S$ and the nodes in layers $2 \cdots L$. The nodes in layer 1 and node $R$ have cannot have an AP.

The common general equations used to derive the probability $P(\exists C A)$ of having a $\mathrm{CA}$ and the probability $P(\exists A P)$ of having an AP in the next steps are:

$$
\begin{aligned}
& P(\exists C A)=1-P(\nexists C A) \\
& P(\nexists A P)=(1-P(\exists C A))^{N-1} \Rightarrow \\
& P(\exists A P)=1-P(\nexists A P)=1-(1-P(\exists C A))^{N-1}
\end{aligned}
$$

- Strict CA method: When a candidate AP $v$ can be chosen as an AP of a node $u$ if the PP of the PP of the node is the same as the $\mathrm{PP}$ of the candidate AP, i.e., if $P P(P P(u))=P P(v)$. An example presenting this case is shown in Figure 1a. Node $S$ and the nodes in layers $2 \cdots L$ have the same number of neighbors, thus the prob- 


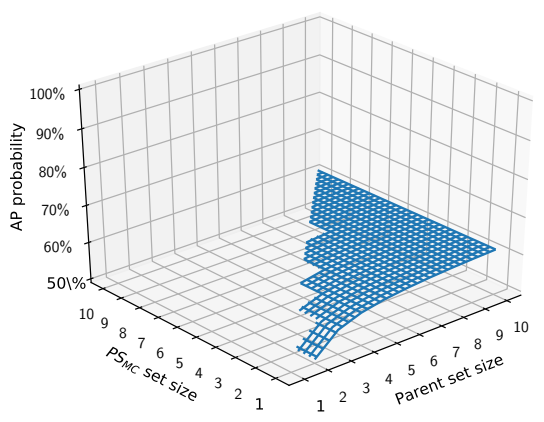

(a) Strict.

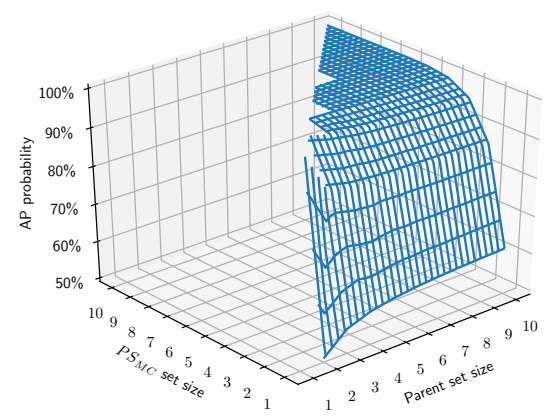

(b) Medium.

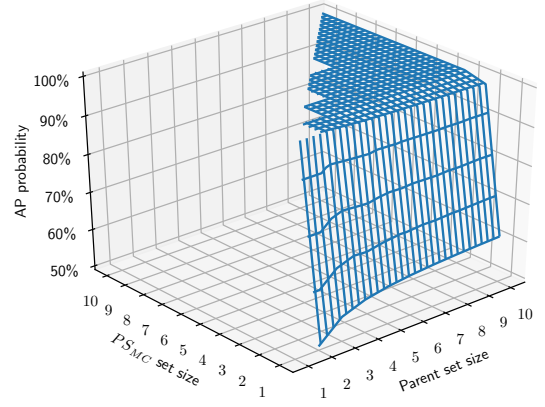

(c) Soft.

Fig. 3: Probability of finding an AP through common ancestors, parametrized over the PS size $N$ and the $P S_{M C}$ set size $M$.

ability of finding an AP is represented by the Equation 2.

$$
P(\exists C A)=\frac{1}{N}
$$

where $P(\exists C A)$ is the probability of having at least one $\mathrm{CA}$ and $P(\exists A P)$ is the probability of having at least one AP (the complement of probability $P(\nexists A P)$ of not being able to find any APs). Given these, the probability of finding an AP is calculated in Equation 3.

$$
P(\exists A P)=1-\left(\frac{N-1}{N}\right)^{N-1}
$$

- Medium CA method: When a candidate AP $v$ can be chosen as an AP of a node $u$ if the PP of the PP of the node is contained in the $P S_{M C}$ of the candidate AP, i.e., if $P P(P P(u)) \in P S_{M C}(v)$. An example presenting this case is shown in Figure $1 \mathrm{~b}$. The probability of having an AP is given by Equation 4 .

$$
P(\exists C A)=\frac{M}{N}
$$

Using Equation 1, we obtain Equation 5.

$$
P(\exists A P)=1-\left(\frac{N-M}{N}\right)^{N-1}
$$

- Soft CA method: When a candidate AP $v$ can be chosen as an AP of a node $u$ if the $P S_{M C}$ of the PP of the node has any overlap with the $P S_{M C}$ of the candidate AP, i.e., if $P S_{M C}(P P(u)) \cap P S_{M C}(v) \neq \varnothing$. An example presenting this case is shown in Figure 1c.

In Equation 6 the probability $P(\exists C A)$ of obtaining a CA depends on the probability of having a common node in the two $P S_{M C}$ parent sets via the PP and via the candidate AP.

$$
\begin{aligned}
& P(\nexists C A)=\frac{\left(\begin{array}{l}
N \\
M
\end{array}\right) \cdot\left(\begin{array}{c}
N-M \\
M
\end{array}\right)}{\left(\begin{array}{c}
N \\
M
\end{array}\right) \cdot\left(\begin{array}{c}
N \\
M
\end{array}\right)}=\frac{\left(\begin{array}{c}
N-M \\
M
\end{array}\right)}{\left(\begin{array}{c}
N \\
M
\end{array}\right)} \Rightarrow \\
& P(\exists C A)=1-\frac{\left(\begin{array}{c}
N-M \\
M
\end{array}\right)}{\left(\begin{array}{c}
N \\
M
\end{array}\right)}
\end{aligned}
$$

Finally, using Equation 1, the probability of finding an AP is represented by the Equation 7 .

$$
P(\exists A P)=1-\left[\frac{\left(\begin{array}{c}
N-M \\
M
\end{array}\right)}{\left(\begin{array}{l}
N \\
M
\end{array}\right)}\right]^{N-1}
$$

To make these results more concrete, the probability of finding an AP through common ancestors with the three methods, parametrized over the PS size $N$ and the $P S_{M C}$ set size $M$ is shown in Figure 3. Analyzing the values of Figure 3a (Strict CA), it can be observed that having a greater number of parents increases the probability of obtaining an AP, reaching its maximum when it has 5 parents, with a probability close to $60 \%$. Since this method does not use the Parent Set information, the size $M$ of the $P S_{M C}$ does not affect the probability.

On the other hand, in the case of Figure 3b (Medium CA) and Figure 3c (Soft CA) respectively, it can be observed that their behavior is similar. This is because they are both directly influenced by the number $M$ of addresses in the PS extension in the NSA MC. However, the Soft CA has a higher probability value than Medium CA for the same parameters, since it uses the intersection between the two $P S_{M C}$ sets, while Medium CA uses just one parent (the $\mathrm{PP}$ ) from one of the two $P S_{M C}$ sets intersected with one $P S_{M C}$ set. Due to this, the Soft CA reaches $100 \%$ since there are values of $N, M$ with guaranteed non-empty intersection.

\section{Performance Evaluation}

\section{A. Simulation setup}

To obtain a realistic evaluation of performance, we used the COOJA simulator developed as part of Contiki [2]. The environment in which the simulations were executed has the following characteristics:

- The topology (shown in Figure 2) consists of a matrix of 5x6 nodes, with 5 layers of 6 nodes. At the upper and lower ends of the matrix, there is the source node $S$ and the DODAG root $R$.

- Each node in layer $i$ has 6 parents in layer $i-1$ with the exception of the nodes in layer 1 , which only have the root $(R)$ node in their parent set. 


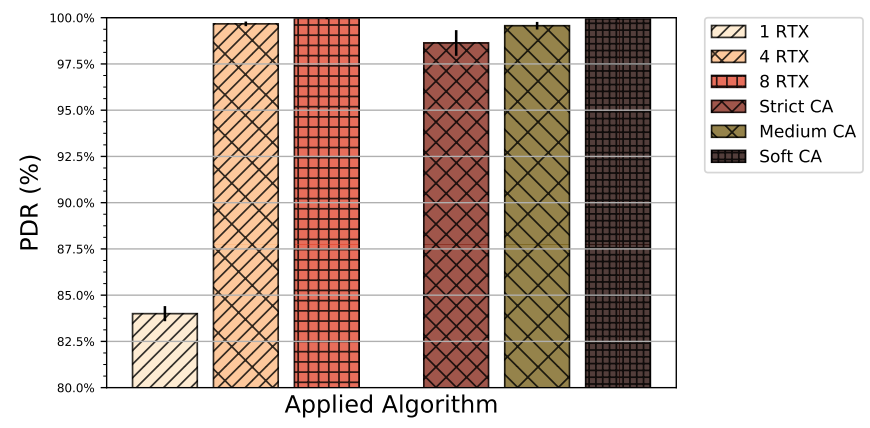

Fig. 4: Average Packet Delivery Ratio (PDR).

\begin{tabular}{lc|lc}
\hline \hline \multicolumn{2}{c|}{ Simulation } & \multicolumn{2}{c}{ Topology } \\
\hline Duration & until 1000 pkts & Topology & Multi-hop \\
Data traffic & $1 p k t / 15 \mathrm{sec}$ & № of nodes & 32 \\
Routing & RPL & № of layers $(L)$ & 5 \\
Parent set size $(N)$ & 6 & № of sources & 1 \\
$P S_{M C}$ size $(M)$ & 3 & Link quality & $70 \%-100 \%$ \\
\hline \hline TSCH & Single-path & Multi-path \\
\hline Scheduling & Centralized & Centralized \\
EB period & $4 \mathrm{sec}$ & $4 \mathrm{sec}$ \\
Timeslot length & $10 \mathrm{~ms}$ & $10 \mathrm{~ms}$ \\
Slotframe length & 297 Timeslots & 297 Timeslots \\
№ of channels & 1 & 1 \\
№ of RTX & 1 RTX, 4 RTX, 8 RTX & 1 RTX \\
\hline
\end{tabular}

TABLE I: Simulation parameters.

- Simulations with single-path RPL were made with different values of MAC RTXs to evaluate the trade-off between reliability and energy consumption.

Table I contains the detailed parameters used during the simulations.

\section{B. Simulation results}

In terms of PDR, the probability of having an AP directly affects performance, since a greater number of forwarding nodes leads to a greater number of copies of packets, which in turn increases reliability. The results obtained from the simulations illustrate this in Figure 4. More specifically, in the case of single-path, the PDR is $82.7 \%$, since the reliability depends only on the PP. A significant improvement to $97.32 \%$ PDR can be achieved with Strict CA, due to the use of PRE, i.e., multiple routes.

However, with Strict CA the availability of an AP has a relatively low probability of $59.8 \%$. As a result, when this does not happen only a single route is available, reducing reliability.

In the case of Medium CA, the PDR obtained is $99.66 \%$. Compared to Strict CA, the triangular pattern is more flexible, since the selection of the AP will depend on whether it has within its set of parents the preferred grandparent of the node. Furthermore, the PDR value of Soft CA is $99.98 \%$ due to its even more relaxed criteria for selecting an AP.

In terms of delay and jitter, as show in Figure 5 and Figure 6, both the delay and jitter achieved by the CA algorithms are very competitive with the single-path algorithms. Jitter especially is very low in all the CA algorithms, being virtually the same as the 1 RTX single-path algorithm.

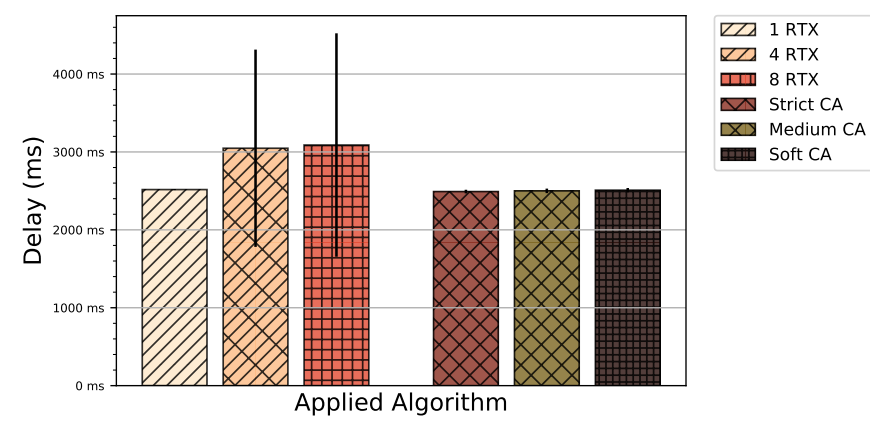

Fig. 5: Average Delay and Jitter per packet.

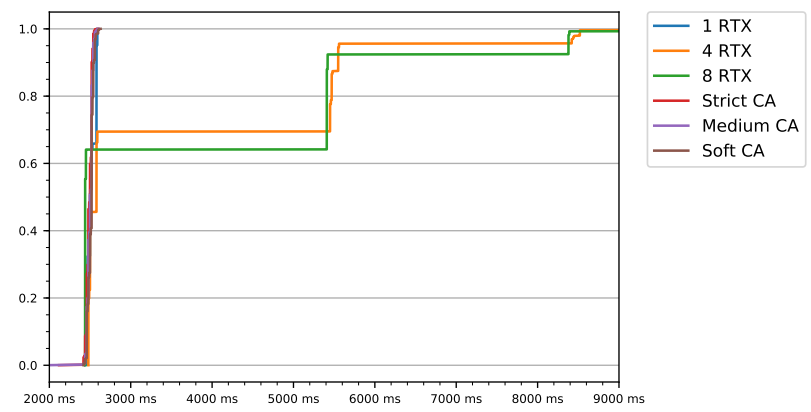

Fig. 6: Cumulative Distribution Function of Delay.

In terms of traffic overhead due to replication, the number of packet copies forwarded per packet, shown in Figure 7a, depends on how strict the selection is, as in the case of PDR. It can be seen that in the single-path case, the value is slightly higher than the number of hops that a packet has to take to reach the root, due to RTXs along that path. On the other hand, the CA algorithms generate approximately $235 \%-400 \%$ more packet copies as part of the multi-path replication process. A similar pattern appears in the results for the number of traversed nodes per packet, shown in Figure $7 \mathrm{~b}$, where the multi-path CA algorithms lead to the usage of more nodes than the single-path ones. In terms of network stability as expressed by the number of DIO packets transmitted, as shown in Figure 7c, the single-path and the CA algorithms show similar results, with a slightly lower number of DIOs transmitted when a higher RTX count is used. DIOs are not affected by replication, but UDP data packets are, and as a result as in Figure 7a, the number of packets transmitted for the CA algorithms is higher than the single-path ones.

Finally, in terms of power consumption, we used the values of the Zolertia Z1 mote ${ }^{1}$, which uses the CC2420 radio transceiver module. Given the values for radio power consumption for the Z1 $\left(P_{T X}=52.2 \mathrm{~mW} @ 3 \mathrm{~V}\right.$, $\left.P_{R X}=56.4 \mathrm{~mW} @ 3 \mathrm{~V}, P_{\text {Idle }}=1.28 \mathrm{~mW} @ 3 \mathrm{~V}\right)$, the energy consumption was calculated as shown in Equation 8:

$$
E(P)=P_{T X} C_{T X}+P_{R X} C_{R X}+P_{\text {Idle }} C_{\text {Idle }}
$$

Figure 8 shows the results obtained during the simulations. It can be seen that the CA algorithms consumed approximately $29 \%-44 \%$ more energy than the single-path algorithms. A

\footnotetext{
${ }^{1}$ Zoletria Z1 datasheet, Rev. C. March 2010.
} 


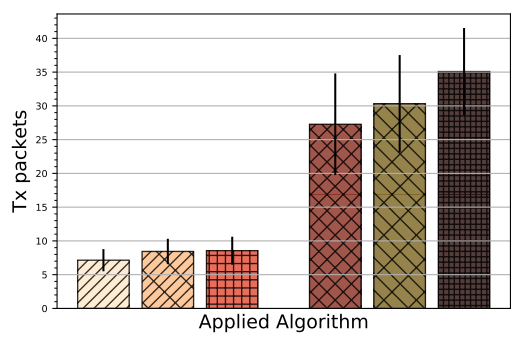

(a) Packet copies forwarded per source packet.

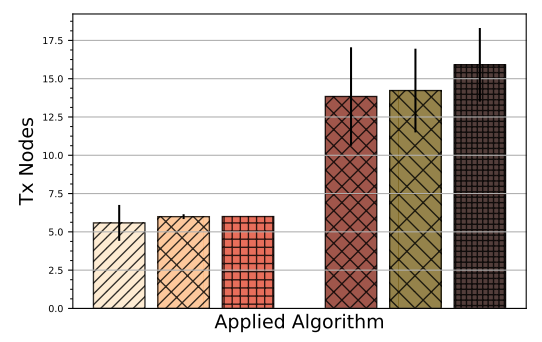

(b) Number of traversed nodes.

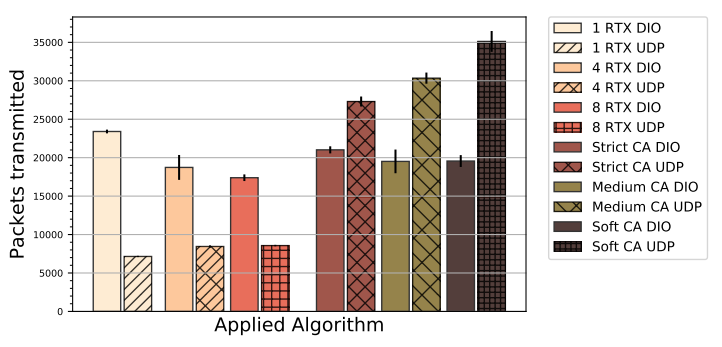

(c) Total DIO and UDP packets.

Fig. 7: Network traffic overhead results.

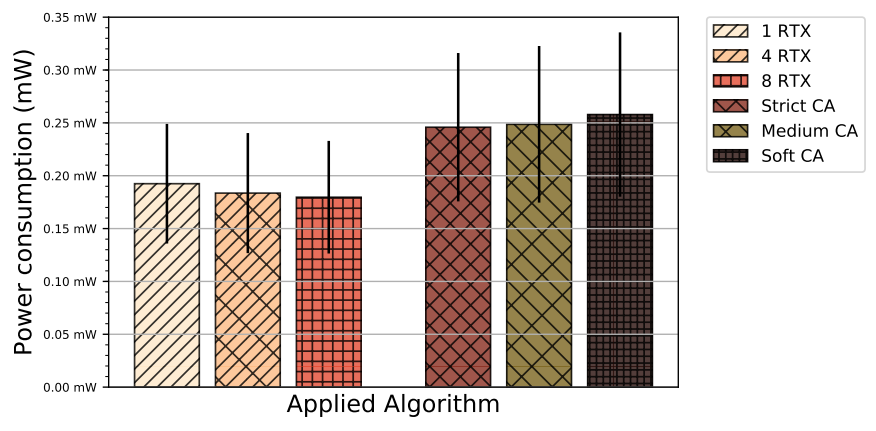

Fig. 8: Average Power Consumption per node.

part of this can be attributed to the slightly smaller number of DIO packets in the single-path case with many RTXs, however the majority of the difference is due to the additional number of packets transmitted.

Although the overall network power consumption is higher for our algorithms in comparison to the default single-path algorithms, given the focus on low delay and jitter and high PDR, the trade-off presented may be reasonable. Comparing the two best options for this use case, single-path with 8 RTXs and Soft CA, in exchange for $44 \%$ additional power consumption, Soft CA offers virtually the same high PDR and in addition $18.75 \%$ lower delay. Even more importantly, it produces much lower jitter: the jitter with the single-path algorithm is $5200 \%$ higher than the Soft CA. The centralized TSCH schedule used in the simulations allows two upstream data transmissions per slotframe, therefore as the number of RTXs increases, more slotframes are required, in turn increasing delay and jitter. The CA algorithms always use just one slotframe. The use of a schedule with more upstream data transmissions would benefit the jitter for the single-path algorithms but would also increase the delay as well. In terms of PDR, a single-path algorithm improves considerably as the RTXs increase, while PRE improves as its Common Ancestor selection becomes less strict.

\section{CONCLUSIONS}

For the topology analyzed, the minimum probability of having an AP is $60 \%$, increasing as the number of parents increases and as the number of addresses contained in the PS extension of the NSA metric in DIO messages increases. We identified that although the overall network power consumption is higher for our algorithms in comparison to the default single-path algorithms, the trade-off presented is useful for the intended deterministic network performance applications. Achieving very low jitter is extremely important since it allows calculating the maximum delay over a multihop networks and thus to obtain predictable performance. Finally, the use of PRE provides an improvement in reliability with a PDR above $95 \%$ and also guarantees a low Jitter.

\section{ACKNOWLEDGEMENTS}

This work was partially performed and supported under the TPI ANR-17-CE10-0007-01 project of the French National Research Agency (ANR).

\section{REFERENCES}

[1] IEEE Standard for Local and Metropolitan Area Networks - Part 15.4: Low-Rate Wireless Personal Area Networks (LR-WPANs) Amendment 1: MAC Sublayer. IEEE Std 802.15.4e-2012 (Amendment to IEEE Std 802.15.4-2011), April 2012.

[2] A. Dunkels, B. Gronvall, and T. Voigt. Contiki - a Lightweight and Flexible Operating System for Tiny Networked Sensors. In Proceedings of the 29th Annual IEEE International Conference on Local Computer Networks (LCN), 2004.

[3] O. Iova, F. Theoleyre, and T. Noel. Using multiparent routing in RPL to increase the stability and the lifetime of the network. Ad Hoc Networks, 29:45-62, 2015.

[4] R. A. Koutsiamanis, G. Z. Papadopoulos, X. Fafoutis, J. M. Del Fiore, P. Thubert, and N. Montavont. From Best-Effort to Deterministic Packet Delivery for Wireless Industrial IoT Networks. IEEE Transactions on Industrial Informatics, 14:4468 - 4480, 2018.

[5] R. A. Koutsiamanis, G. Z. Papadopoulos, N. Montavont, and P. Thubert. RPL DAG Metric Container (MC) Node State and Attribute (NSA) object type extension. draft-ietf-roll-nsa-extension-00, IETF, December 2018.

[6] Q. Le, T. Ngo-Quynh, and T. Magedanz. RPL-based multipath Routing Protocols for Internet of Things on Wireless Sensor Networks. In Proceedings of the International Conference on Advanced Technologies for Communications (ATC'14), 2014.

[7] P. Minet, I. Khoufi, and A. Laouiti. Increasing Reliability of a TSCH Network for the Industry 4.0. In Proceedings of the 16th IEEE International Symposium on Network Computing and Applications (NCA), 2017.

[8] G. Z. Papadopoulos, T. Matsui, P. Thubert, G. Texier, T. Watteyne, and N. Montavont. Leapfrog Collaboration: Toward Determinism and Predictability in Industrial-IoT applications. In Proceedings of the IEEE International Conference on Communications (ICC), 2017.

[9] G. Z. Papadopoulos, N. Montavont, and P. Thubert. Exploiting Packet Replication and Elimination in Complex Tracks in 6TiSCH LLNs. draft-papadopoulos-6tisch-pre-reqs-02, IETF, July 2018.

[10] T. Watteyne, M. Palattella, and L. Grieco. Using IEEE 802.15.4e Time-Slotted Channel Hopping (TSCH) in the Internet of Things (IoT): Problem Statement. RFC 7554, 2015.

[11] T. Winter, P. Thubert, A. Brandt, J. Hui, R. Kelsey, P. Levis, K. Pister, R. Struik, JP. Vasseur, and Alexander R. RPL: IPv6 Routing Protocol for Low-Power and Lossy Networks. IETF RFC 6550, 2012. 